\title{
Programa de Mentoria do Curso de Medicina da Universidade Federal do Rio Grande do Norte: Atividades Integrativas em Foco
}

\author{
The Universidade Federal do Rio Grande do Norte Medicine \\ Course Mentoring Program: Integrative Activities in Focus
}

\section{PALAVRAS-CHAVE}

- Educação Médica.

- Educação em Saúde.

- Mentoria.

- Estudante Universitário.

\author{
Simone da Nóbrega Tomaz Moreira ${ }^{I}$ (iD \\ Iana Ciara Santos de Albuquerque ${ }^{I}$ (D) \\ Francisco Edilson Leite Pinto Junior ${ }^{I}$ (D) \\ Alexandre Henrique Bezerra Gomes ${ }^{I}$ iD
}

\section{RESUMO}

Introdução: Ao ingressarem no curso de Medicina, os estudantes se deparam com desafios inerentes à formação que podem ser geradores de estresse e ansiedade, comprometendo seu bem-estar e desempenho acadêmico. Diante disso, o curso de Medicina da Universidade Federal do Rio Grande do Norte (UFRN) implantou em 2015 um programa de mentoria que se propõe a contribuir para o desenvolvimento profissional e pessoal do estudante, adotando-se como um diferencial a realização de atividades integrativas. Método: Participam desse programa 25 professores do curso de Medicina da UFRN, que atuam como mentores, 25 monitores ou mentores juniores, que têm o papel de intermediar a comunicação entre os participantes, como também auxiliar no planejamento e desenvolvimento das atividades, e 317 alunos de diversos períodos do curso médico. Além dos encontros mensais regulares, no final de cada semestre, os mentores e mentores juniores organizam a atividade integrativa que agrega todos os discentes e docentes do programa e possibilitam a construção de uma relação mais próxima entre mentores e mentorandos, bem como contribuem para a estruturação de um ambiente universitário mais acolhedor e equânime. Resultado: Em avaliação on-line sobre o programa, os alunos destacaram a "troca de experiências, de sugestões e a ajuda no curso sobre diversos temas" e "adquirir experiência de um profissional experiente" como principais motivações para participar da atividade. Contudo, enfatizaram a falta de tempo para conciliar os encontros com as demais atividades acadêmicas como principal obstáculo para participar do programa. Com a pandemia da Covid-19, mantiveram-se as atividades do programa no modelo on-line, e obteve-se importante engajamento dos participantes, o que representou uma estratégia de enfrentamento do isolamento social e de promoção da saúde mental para os estudantes. Conclusão: Observamos, a partir do engajamento e dos feedbacks recebidos, que o programa, apesar de apresentar alguns desafios, vem se configurando como uma iniciativa capaz de transformar as relações interpessoais entre discentes e mentores, ao promover a integração entre alunos dos diferentes períodos do curso e criar um ambiente favorável ao diálogo e à construção do conhecimento. 


\section{KEYWORDS}

- Medical Education.

- Health Education.

- Mentoring.

- University Student.

\section{ABSTRACT}

Introduction: Upon starting their medical course, students are faced with challenges inherent to training, which can generate stress and anxiety, compromising their well-being and academic performance. In view of this, in 2015 the Medicine course at the Federal University of Rio Grande do Norte (UFRN) implemented a Mentoring Program, which aims to contribute to students' professional and personal development, focusing on integrative activities to strengthen bonds between the participants. Method: This program involves the participation of 25 professors from the UFRN Medicine course, who act as mentors, 25 monitors or mentors, who have the role of mediating communication between participants and assisting in the planning and development of activities; and about 317 students and various academic periods of the medical course. In addition to the regular monthly meetings, at the end of each semester, mentors and junior mentors organize the integrative activity that brings together all the students and teachers of the program and have stimulated a closer relationship between mentors and mentees, as well as contributing toward the structuring of a more welcoming and equitable university environment. Result: In an online assessment of the program, students highlighted the "exchange of experiences, suggestions and help with the course on various topics" and "acquiring experience from an experienced professional" as the main motivations for participating in the activity. On the other hand, they emphasized the lack of time to reconcile meetings with other academic activities as the main obstacle to participating in the program. With the onset of the Covid-19 pandemic, the program activities continued online and important engagement was achieved among the participants, thus constituting a strategy to face social isolation and promote the mental health of undergraduate medical students. Conclusion: From the engagement and the feedback received, it was observed that the program, despite presenting some challenges, represents an initiative capable of transforming interpersonal relationships between students and mentors, promoting integration between students from different academic periods of the course and creating a favourable environment for dialogue and knowledge construction.

Recebido em 14/4/20

Aceito em 26/9/20

\section{INTRODUÇÃO}

A inserção do estudante no contexto universitário é um momento marcado, muitas vezes, pela insegurança e ansiedade ${ }^{1}$. Fatores de estresse, como carga horária extensa, grande competitividade entre os colegas, novas exigências cognitivas de estudo e de desempenho, dificuldades na gestão do tempo e privação do sono, podem influenciar negativamente o desempenho dos estudantes em suas atividades ${ }^{2,3}$.

No curso médico, a aproximação dos estudantes a situações de dor, sofrimento e morte tende a contribuir negativamente para a saúde mental deles. Muitos vivenciam de forma solitária esses momentos, uma vez que a racionalidade científica, presente na formação médica, não valoriza aspectos subjetivos do cotidiano acadêmico ${ }^{2}$.

Diante disso, as escolas médicas têm procurado desenvolver estratégias de suporte aos estudantes, de modo a promover espaços de reflexão e acolhimento aos sentimentos e às vivências derivados do processo de formação.

Os programas de mentoria se inserem nesse contexto efetivando-se como uma modalidade de relação de ajuda em que uma pessoa experiente e empática, o mentor, orienta um jovem iniciante, o mentorado, em seu desenvolvimento pessoal e profissional ${ }^{3}$. Mentores podem ser modelos positivos que auxiliarão os alunos na construção da sua identidade profissional, minimizando os estresses advindos do processo de desenvolvimento do vir-a-ser-médico.

Por meio de uma interação baseada na confiança, os programas de mentoria têm o potencial de contribuir na construção de um espaço seguro de partilha de sentimentos, angústias e preocupações ${ }^{4}$, de modo a ajudar os estudantes com problemas mais simples do cotidiano, detectar, precocemente, dificuldades mais significativas e providenciar encaminhamentos para atendimentos especializados nos serviços de apoio psicológico e/ou psiquiátrico ${ }^{5}$.

Cada vez mais, destaca-se a importância dos programas de mentoria na formação integral do estudante de Medicina, na medida em que valorizam e reconhecem os componentes afetivo, ético, social e cognitivo do processo educacional. Ao mediar a busca de soluções para problemas de natureza acadêmica, pessoal e social, a mentoria unifica as duas facetas da educação, instrutiva e formativa, e promove um equilíbrio entre o desenvolvimento pessoal do aluno e sua formação profissional. A mentoria tem sido reconhecida como muito importante para atingir objetivos de carreira e, no campo da saúde mental, contribui para o desenvolvimento de autoconfiança dos estudantes, a redução de estresse e o aumento da sensação de bem-estar deles ao ampliar a sua rede de apoio na instituição ${ }^{6-9}$.

Programas de mentoria podem adotar diversos modelos de acordo com as necessidades e a realidade de cada instituição ${ }^{10}$, estabelecendose como atividade curricular ou de extensão, obrigatória ou voluntária, com grupos homogêneos ou heterogêneos de alunos. O importante é que possam estar em contínua avaliação e aprimoramento, de modo a contribuir para o desenvolvimento pessoal e profissional do estudante e colaborar para um caminho de sucesso no curso e na profissão.

Este relato traz a experiência de um programa de mentoria desenvolvido no curso de Medicina da Universidade Federal do Rio Grande do Norte (UFRN), descrevendo sua implementação e seu desenvolvimento ao longo do tempo. Em especial, busca destacar a introdução, para além dos encontros regulares entre cada mentor e seu grupo de alunos, de novas atividades, denominadas "atividades integrativas", envolvendo ao mesmo tempo, e de forma criativa, todos os participantes do programa.

REVISTA BRASILEIRA DE EDUCAÇÃO MÉDICA

2 44 (4) : e169; 2020 


\section{O PROGRAMA DE MENTORIA NA UFRN}

Implementação

No final de 2014, sob a orientação da coordenação do curso de Medicina da UFRN, foi realizada uma oficina com o propósito de delinear, com os professores, as bases para a instituição de um programa de mentoria alinhadas às necessidades de seus alunos. Essa oficina foi conduzida pela coordenadora do Programa de Mentoria da Faculdade de Medicina da Universidade de São Paulo (FMUSP), a psicóloga e doutora Patrícia Bellodi.

Nessa reunião, abordaram-se os objetivos do programa sob a ótica de um processo em que uma pessoa experiente e empática (o mentor) guia outro indivíduo (geralmente mais jovem - o mentorado) no desenvolvimento e reexame de suas próprias ideias, aprendizado e desenvolvimento pessoal e profissional. O mentor, que muitas vezes (mas não necessariamente) trabalha na mesma organização ou no mesmo campo do mentorado, consegue isso ouvindo ou conversando, a partir do estabelecimento de uma relação de confiança com o mentorado ${ }^{6}$.

Nesse sentido, é imprescindível que professor-mentor seja médico de formação ou docente ou preceptor do curso. Estabeleceram-se também os atributos necessários à figura do mentor: desejo de aprender e se aprofundar sobre a metodologia aplicada na mentoria; disponibilidade para encontros mensais e individualizados; empatia para desenvolver um bom relacionamento com os alunos a fim de compreendê-los nas dificuldades vividas no processo da formação; criatividade; ser alguém capaz de motivá-los para os encontros mensais; acessível; e conhecedor da estrutura curricular do curso. Dos professores presentes naquele momento, 29 manifestaram interesse em participar do programa.

Dessa maneira, adotou-se a modalidade de mentoria em grupo, sendo este formado por um mentor (profissional capacitado de maneira adequada para essa tarefa) e mentorados (alunos de Medicina, no mínimo oito, dos diferentes períodos do curso). Acredita-se que grupos heterogêneos, com alunos de diferentes períodos do curso, contribuem para a troca de experiência, ampliando a percepção deles sobre o curso, como também permitem ao mentor compreender o curso de forma mais longitudinal, conhecendo os desafios dos discentes em cada período do curso ${ }^{11}$.

Ainda nessa reunião, foi acordado com a Pró-Reitoria de Graduação (Prograd) que os grupos teriam reuniões mensais, em um horário protegido. Portanto, nas últimas sextas-feiras do mês, no final da tarde, durante duas horas, as atividades do curso deveriam ser suspensas para que os alunos pudessem participar das reuniões dos grupos de mentoria. Por fim, esse componente curricular foi vinculado à coordenação do curso de Medicina, sendo coordenado por uma psicóloga, professora do eixo ético humanístico do curso, responsável pela organização dos grupos de mentoria e das atividades do programa.

\section{Desenvolvimento}

Em seu primeiro ano de funcionamento, 2015, as atividades do programa foram desenvolvidas como voluntárias, sendo as horas a ele dedicadas aproveitadas como atividade complementar ao curso. A partir do primeiro semestre de 2016, o programa passou a funcionar como disciplina optativa, com o objetivo de computar formalmente a carga horária tanto para discentes quanto para docentes.

Desde o início do programa, os grupos de mentoria reúnem-se para discussão de diferentes temáticas referentes à formação médica. Essas temáticas são escolhidas livremente por cada grupo, a partir dos interesses e das necessidades de seus componentes e aptidões do mentor que tem o papel de facilitar as discussões, levantando questionamentos e reflexões. Esse enquadre foi definido com o propósito de promover o diálogo para um meio equânime, em que os alunos possam falar livremente, se expressar em um ambiente seguro e livre de julgamentos, conhecendo a si e os outros ${ }^{5}$.

Em 2018, o programa ofereceu 25 vagas para alunos monitores, os quais foram denominados mentores juniores, cujo papel era colaborar nas atividades de planejamento em parceria com coordenação do programa. Havia também a expectativa de que esses mentores juniores incentivassem o engajamento dos mentores e contribuíssem para um diálogo mais horizontal entre estes e os alunos. Para desempenhar essa função, foi definido como pré-requisito ter cursado, pelo menos, dois semestres letivos da disciplina optativa de Mentoria.

O programa foi muito beneficiado com participação desses monitores que, por iniciativa própria, organizaram-se em quatro comissões: 1. temas e metodologias, 2. atividades integrativas, 3. mídias sociais e 4 . pesquisa. Tais comissões passaram a se reunir mensalmente para a organização de estratégias que pudessem aprimorar o programa. Os mentores juniores colaboram no planejamento das atividades do semestre, refletem sobre possíveis temáticas que poderão ser discutidas nos grupos e, especialmente, ajudam a organizar as atividades integrativas, a seguir descritas. Desenvolveram o site do programa e são responsáveis pela divulgação, por meio das mídias sociais, das atividades e dos eventos. Por fim, mas não menos importante, colaboram também na avaliação das atividades desenvolvidas.

\section{AS ATIVIDADES INTEGRATIVAS}

A partir do segundo semestre de 2015, com o objetivo de ampliar a interação entre os integrantes do programa, além promover os encontros mensais regulares dos grupos de mentoria, foram criadas as chamadas "atividades integrativas". Nessas atividades, de maneira mais descontraída e lúdica, todos os grupos do programa são envolvidos em um evento coletivo, ao final de cada semestre (Quadro 1).

A primeira atividade integrativa realizada foi uma mesa-redonda sobre especialidades médicas. $\mathrm{Na}$ ocasião, médicos de diferentes áreas compartilharam seus processos de escolha da especialidade, a rotina de trabalho e as atividades desenvolvidas na especialidade. Além disso, responderam às perguntas dos estudantes sobre qualidade de vida, mercado de trabalho na área, entre outras. Essa atividade foi repetida mais uma vez por solicitação dos estudantes.

Outra atividade foi o Consultório Filosófico (CF), realizada em conjunto com o Departamento de Filosofia da UFRN. Essa atividade interativa objetivou propiciar reflexões filosóficas sobre alguns temas que permeiam a vida humana e contribuir para a divulgação de conteúdos filosóficos para o público leigo em filosofia. O CF foi realizado em duas etapas. Na primeira, apresentaram-se aos pacientes (estudantes de Medicina) seis frases filosóficas para que eles escolhessem aquela com que mais se identificavam. A segunda foi a "consulta" em si, na qual o paciente (estudante), que era atendido por "médico-filósofo", falava sobre as questões existenciais que o afetavam a partir das frases escolhidas. $\mathrm{O}$ paciente (estudante) recebia então uma "receita" com o "diagnóstico" da "doença" e da medicação prescrita, que era a indicação da leitura de uma obra filosófica.

REVISTA BRASILEIRA DE EDUCAÇ̄̃̃ MÉDICA

3 44(4) : e169; 2020 


\begin{tabular}{|c|c|}
\hline & $\begin{array}{l}\text { Quadro 1 } \\
\text { Atividades integrativas do Programa de Mentoria }\end{array}$ \\
\hline Semestre letivo & Atividade \\
\hline 2015.2 e 2016.1 & $\begin{array}{l}\text { Mesa-redonda: especialidades médicas - A atividade visou abordar pontos de curiosidade dos alunos a respeito das diversas } \\
\text { especialidades médicas, do tempo da residência, da remuneração e do mercado de trabalho. }\end{array}$ \\
\hline 2016.2 e 2019.2 & $\begin{array}{l}\text { Gincana Mentoring - Realizada em um parque de preservação ambiental, a ação buscou despertar nos participantes preceitos relativos } \\
\text { ao trabalho em equipe e à consciência social, angariando doações de alimentos para comunidades em situação de vulnerabilidade social. }\end{array}$ \\
\hline 2017.1 & $\begin{array}{l}\text { Consultório Filosófico - Em conjunto com alunos do curso de Filosofia, a atividade buscou fomentar reflexões para os estudantes do } \\
\text { curso médico, a respeito questões existenciais da vida humana. }\end{array}$ \\
\hline 2017.2 & $\begin{array}{l}\text { CineMentoring - A atividade fomentou a discussão de uma obra cinematográfica em um ambiente integrativo para os grupos tutoriais } \\
\text { em conjunto, estreitando o vínculo entre os membros da disciplina. }\end{array}$ \\
\hline 2018.1 e 2019.1 & $\begin{array}{l}\text { Arraiá do Mentoring - Ação realizada em duas edições, sob a perspectiva regional da festa junina, a fim de integrar os alunos dos } \\
\text { diversos grupos mentoriais e promover um ambiente seguro de descontração para seus membros. }\end{array}$ \\
\hline 2018.2 & $\begin{array}{l}\text { Mentoring Solidário - A atividade solidária proporcionou aos alunos o contato com a realidade social de uma comunidade em } \\
\text { situação de vulnerabilidade, oferecendo, na época dos festejos natalinos, a doação de presentes para as crianças dessa população. }\end{array}$ \\
\hline
\end{tabular}

Fonte: Elaborado pelos autores.

A Gincana Mentoring, outra atividade integrativa realizada, demandou que os membros de cada grupo de mentoria se organizassem para cumprir um conjunto de tarefas estabelecidas ao longo de um semestre. A consolidação da atividade ocorreu em um parque/área de proteção ambiental da cidade, com o propósito de estimular o trabalho em equipe e o gerenciamento de pessoas, recursos e tempo. Nessa ação, uma das tarefas foi angariar alimentos para doação em uma comunidade em situação de vulnerabilidade social, atribuindo-se, assim, valor social à atividade.

Com o mesmo propósito, outra atividade integrativa de grande destaque foi o Mentoring Solidário. Nessa atividade, cada grupo de mentoria "apadrinhou" uma criança de uma comunidade em situação de vulnerabilidade social da cidade de Natal. Cada criança recebeu kits escolares em um encontro repleto de alegria e afeto.

Entre as propostas de integração realizadas, pode-se também destacar o CineMentoring, realizado no Auditório da Biblioteca Central do câmpus, quando os grupos de mentoria, em um momento de confraternização, assistiram em conjunto ao filme Hipócrates ${ }^{12}$, que retrata o cotidiano de um residente de medicina. Após isso, ocorreu uma discussão sobre questões pertinentes à educação médica, numa troca de experiência entre os participantes.

Outra atividade integrativa de grande prestígio no Programa de Mentoria foi o Arraiá do Mentoring, realizada também em duas edições. Essa atividade se caracterizou pela regionalidade da temática das festas juninas, atrelada a comidas típicas, quadrilha e um ambiente descontraído para estimular a integração entre os discentes e docentes do programa.

Em todas as atividades integrativas, é importante ressaltar que os monitores exerceram papéis de liderança, pois eles as planejaram, estimularam o envolvimento de todos os grupos e inseriram no programa benefícios da mentoria de pares $^{13}$, como aprimoramento de habilidades de comunicação e autogestão, resolução de conflitos e gestão de recursos e de pessoas.

\section{FEEDBACK DOS ALUNOS SOBRE O PROGRAMA}

Para o planejamento das atividades de 2019, incentivou-se, no final do ano, que os alunos respondessem a um formulário on-line de avaliação do programa construído pelos mentores juniores. Nesse formulário, havia as seguintes perguntas:
- $\quad$ O que motiva você a participar da mentoria?

- $\quad$ O que desmotiva você a participar da mentoria?

- Quais temáticas você gostaria de abordar nas discussões?

- Você possui sugestões para otimizar nosso modelo?

$\mathrm{Na}$ vigência do planejamento das atividades do primeiro semestre de 2019, embora o retorno ao pedido de feedback tenha sido pequeno - obteve-se uma taxa de resposta de 18,9\%, isto é, 60 alunos do total de matriculados no programa $(n=317)$ retornaram aos pesquisadores o questionário preenchido -, uma análise qualitativa das respostas mostrou que, em relação à motivação dos alunos em participar das atividades propostas, por exemplo, os relatos foram positivos:

Os papos descontraídos que envolvem gente como a gente, na mesma luta e com algumas mesmas dificuldades, a troca de experiências, de sugestões e a ajuda no curso sobre diversos temas e dúvidas, além do contato com um profissional que já vivenciou boa parte disso tudo.

A oportunidade de conversar sobre diversos temas, tendo visões diversas. Além de adquirir experiência com a minha mentora! Gosto de saber da vida dela, o que faz, como faz, como foi a universidade, o que ela pensa. A amo!

Variar a rotina com uma atividade que descontrai é interessante. É um momento importante que propicia contato com outros estudantes de modo diferente.

A oportunidade de agregar conhecimentos relacionados à minha futura profissão que não são discutidos em sala de aula, geralmente (como especialidades, oportunidades de emprego, gastos pós-formatura...).

Por sua vez, os principais pontos que desmotivam a participação dos alunos nas atividades propostas giraram em torno da falta de tempo e dos horários das reuniões: 
Preocupação com demais compromissos e a dúvida se valeria a pena comparecer ao encontro com tanta coisa pra fazer.

Não tenho aula na sexta à tarde, então às vezes falta disposição pra ficar na universidade a tarde inteira "ocioso" pra esperar a hora do encontro. Como moro longe, só vou pra casa quando acabam todas as minhas atividades acadêmicas.

Em relação às temáticas mais relevantes para as reuniões e atividades integrativas (Tabela 1), os temas mais citados foram: especialidades médicas $(76,6 \%)$, saúde mental $(61,6 \%)$, dificuldades no curso de Medicina (61,6\%) e estratégias de estudo (56,6\%). Já a maioria das sugestões dos alunos consistiu em realizar as reuniões de mentoria em locais extrauniversitários, de modo a obter um ambiente mais acolhedor e informal para os encontros, como cafés e parques da cidade. Dessa maneira, os alunos teriam maior liberdade para se expressar, sem considerar aquela atividade apenas como um componente curricular, mas sim um espaço de acolhimento para uma discussão descontraída entre amigos.

\section{A MENTORIA DURANTE A PANDEMIA}

Mesmo com a suspensão das atividades presenciais em todas as universidades brasileiras por conta da pandemia causada pela coronavirus disease 2019 (Covid-19), as atividades da mentoria na UFRN tiveram continuidade. Por meio de aplicativos de videoconferência, embora com periodicidade variada e dificuldades de acesso de alguns alunos, os encontros seguiram de forma on-line.

As atividades da mentoria vêm sendo utilizadas como estratégia de enfrentamento do isolamento social e de promoção da saúde mental para os estudantes do curso de Medicina. Nesse cenário, merece destaque a iniciativa de um dos grupos em discutir o filme $O$ poço ${ }^{14}$, comparando-o com a atual situação que vivemos. Tal qual o cenário do filme, em que todos estão no mesmo "poço", faltando "solidariedade espontânea", assim estamos nós diante da virulência do coronavírus.

Outra atividade a se destacar foi o Desafio Mentoring, proposta pelos mentores juniores, visando à proximidade afetiva e social entre os alunos do curso, apesar do distanciamento físico. Assim, em cada semana, um desafio é lançado com uma temática definida para partilha de experiências, dicas e ideias. Nessa proposta, mentores e mentorados participam por meio de suas redes sociais, compartilhando suas atividades diárias realizadas na quarentena, como dicas de filmes, livros, estratégias para lidar com o estresse advindo da pandemia, entre outras questões.

Por fim, uma nova estratégia, denominada "Sala de Conversa", vem sendo implementada como alternativa on-line às atividades integrativas. Nessa sala virtual, convidados especiais abordam temáticas selecionadas previamente, relevantes ao período da pandemia e à educação médica, como telemedicina e marketing digital aplicado à carreira médica.

\section{REFLEXÕES SOBRE A EXPERIÊNCIA}

Atualmente, mais de 50,0\% (317/620) dos alunos do curso de Medicina estão inscritos no Programa de Mentoria. Podemos inferir então que existe um engajamento positivo com relação a participação desses alunos nas atividades propostas. Além disso, a partir de alguns feedbacks deles recebidos, observamos que esses mentorados sentemse acolhidos, pertencentes a um grupo, no qual podem falar sobre as

\begin{tabular}{|ccc|} 
Tabela 1 & \\
Frequência de resposta dos 60 alunos em relação à relevância das \\
temáticas para os encontros da mentoria \\
\hline Temáticas & $\mathbf{n}$ & $\%$ \\
\hline Especialidades médicas & 46 & 76,6 \\
\hline Dificuldades no curso de Medicina & 37 & 61,6 \\
\hline Saúde mental & 37 & 61,6 \\
\hline Estratégias de estudo & 34 & 56,6 \\
\hline Residência médica & 32 & 53,3 \\
\hline Polêmicas sociais & 21 & 35,0 \\
\hline Angústias pessoais & 21 & 35,0 \\
\hline Discussão de artigos científicos & 20 & 33,3 \\
\hline${ }^{*}$ Outros & 7 & 11,6 \\
\hline
\end{tabular}

${ }^{\star}$ Houve outras temáticas citadas pelos alunos que obtiveram baixa frequência de respostas, logo foram agrupadas na categoria "outros" e compreenderam: qualidade de vida e medicina; atividades extracurriculares; organização do tempo; realidade do médico formado; e iniciação à pesquisa.

Fonte: Elaborada pelos autores.

angústias e os temores relacionados à formação médica. Acreditamos que o programa vem contribuindo para a construção de um ambiente de cuidado, construindo um espaço favorável ao diálogo e ao fortalecimento das relações interpessoais ${ }^{11}$.

Consideramos ainda que o Programa de Mentoria, como iniciativa pioneira no curso de Medicina da UFRN, mostrou relevância ao propor a formação integral do indivíduo como pilar do processo de ensinoaprendizagem. $O$ fato de os alunos e professores terem integração da carga horária, com a inclusão da mentoria como componente curricular, constituiu-se um estímulo para participação no programa. Além disso, demonstra o compromisso da instituição com a proposta do programa e reforça a importância do mentoring para o desenvolvimento integral dos alunos. Acreditamos, portanto, que este tem o potencial de contribuir para uma maior satisfação com a escolha profissional e com as relações interpessoais vivenciadas no ambiente acadêmico.

No entanto, apesar do grande potencial desse programa para o curso de Medicina, temos nos deparado com desafios, principalmente no que se refere à falta de envolvimento de alguns mentores e alunos com a proposta, corroborando outros estudos que discutem os entraves ainda enfrentados pelo programa e sua pouca valorização ${ }^{4,5,8}$. Os mentores, por um lado, manifestam insatisfação com alguns alunos, especialmente em relação à adesão. Para eles, a ausência desses alunos mostra desinteresse, o que os deixa desmotivados. Os estudantes, por outro lado, alegam que, apesar de valorizarem a mentoria, têm muitas atividades durante o curso e, em alguns momentos, não conseguem participar das reuniões.

Além disso, consideramos que alguns mentores ainda precisam compreender a proposta como algo mais relacional, que promova a construção de um ambiente acolhedor e informal para as discussões referentes à formação profissional e pessoal, contribuindo para o desenvolvimento da identidade profissional do futuro médico.

A exposição de tais desafios contribui para pensarmos em estratégias que possibilitem a implementação de melhorias ao programa, de modo que as atividades de mentoria sejam (re)pensadas continuamente,

\begin{tabular}{l|l}
5 & REVISTA BRASILEIRA DE EDUCAÇÃO MÉDICA \\
\hline 44 (4) : e169; 2020
\end{tabular} 
como cenário para significativos ganhos acadêmicos e pessoais para os indivíduos envolvidos.

Outrossim, ressaltamos, nesse cenário, o notório impacto das atividades da mentoria no período de pandemia pela Covid-19, como estratégias de enfrentamento do isolamento social e promoção de saúde mental para os estudantes do curso de Medicina da UFRN, propiciando, assim, um ambiente seguro para dar continuidade aos debates e à orientação, outrora realizados pessoalmente e agora adquirindo também um importante papel social, além do formativo já empregado nas atividades da mentoria.

Dessa forma, percebemos que, apesar de alguns desafios inerentes ao funcionamento do Programa de Mentoria, o vínculo estabelecido entre os membros dos grupos, como também o apoio social, tão importante neste momento de pandemia, tem demostrado a importância desse programa como uma estratégia institucional positiva para o enfrentamento dos desafios advindos desse cenário de pandemia.

\section{REFERÊNCIAS}

1. Cameron D, Dromerick LJ, Ahn J, Dromerick AW. Executive/life coaching for first year medical students : a prospective study. BMC Med Educ. 2019;19:163.

2. Moreira SNT, Vasconcellos RLSS, Heath N. Estresse na formação médica: como lidar com essa realidade? Rev Bras Educ Med. 2015;39(4):558-64.

3. Bellodi PL, Martinho T, Massaroppe B, Martins MA, Santos MAS. Temas para um programa de tutoria em medicina: uma investigação das necessidades dos alunos da FMUSP. Rev Bras Educ Med. 2004;28(2):119-27.

4. Ribeiro MMF, Martins AF, Fidelis GTA, Goulart GC, Molinari LC, Tavares EC. Tutoria em escola médica: avaliação por discentes após seu término e ao final do curso. Rev Bras Educ Med. 2013;37(4):509-14.

5. Martins AF, Bellodi PL. Mentoring: uma vivência de humanização e desenvolvimento no curso médico. Interface Comun Saude Educ. 2016;20(58):715-26.

6. Steven A, Oxley J, Fleming WG. Mentoring for NHS doctors: perceived benefits across the personal-professional interface. J R Soc Med. 2008;101(11):552-7.
7. Sánchez LMM, Carrasco FC, Sierra AVR. La acción tutorial como experiencia educativa para la formación integral de los estudiantes de Medicina. Actual Investig en Educ. 2017;17(3):565-88.

8. Fidelis GTA. Mentoring at the school of Medicine at the Federal University of Minas Gerais: from a dream necessary to the construction. Rev Méd Minas Gerais. 2014;24(4):525-34.

9. Ortega G, Smith C, Pichardo MS, Ramirez A, Soto-Greene M, Sánchez JP. Preparing for an academic career: the significance of mentoring. MedEdPORTAL. 2018;14:10690.

10. Frei E, Stamm M, Buddeberg-Fischer B. Mentoring programs for medical students - a review of the PubMed literature 2000-2008. BMC Med Educ. 2010;10:32.

11. Chaves LJ, Gonçalves ECQ, Ladeira LR, Ribeiro MS, Costa MB, Ramos AAM. A tutoria como estratégia educacional no ensino médico. Rev Bras Educ Med. 2014;38(4):532-41.

12. Lilti T. Hipócrates [filme]. Paris - França: Bonfilm; 2014.

13. Akinla O, Hagan P, Atiomo W. A systematic review of the literature describing the outcomes of near-peer mentoring programs for first year medical students BMC Med Educ. 2018;18:98.

14. Gaztelu-Urrutia G. O poço. Madri - Espanha: Netflix; 2019.

\section{CONTRIBUIÇÃO DOS AUTORES}

Todos os autores trabalharam na sistematização das atividades do Programa de Mentoria, que serviram de base para elaboração deste trabalho, bem como participaram da escrita do manuscrito, do delineamento de sua metodologia, do levantamento bibliográfico e da problematização de seus resultados e desafios.

\section{CONFLITO DE INTERESSES}

Os autores declaram não haver conflito de interesses neste relato.

\section{ENDEREÇO PARA CORRESPONDÊNCIA}

Simone da Nóbrega Tomaz Moreira. Avenida Nilo Peçanha, 620, Petrópolis, Natal, RN, Brasil. CEP: 59012-300.

E-mail: simonetomaz@hotmail.com 